\title{
DÜBLIN
}

Technological University Dublin

ARROW@TU Dublin

2014-11

\section{Shaping the Axial-Ratio Footprint of Crossed-Dipole Antennas}

\author{
Adam Narbudowicz \\ Technological University Dublin, adam.narbudowicz@mydit.ie \\ Max J. Ammann \\ Technological University Dublin, max.ammann@tudublin.ie \\ Janusz Przewocki \\ j.przewocki@gmail.com
}

Follow this and additional works at: https://arrow.tudublin.ie/engscheleart

Part of the Electromagnetics and Photonics Commons

\section{Recommended Citation}

A. Narbudowicz, M. J. Ammann, and J. Przewocki, (2014) Shaping the Axial-Ratio Footprint of CrossedDipole Antennas, LAPC - Loughborough Antennas \& Propagation Conference, Loughborough, UK, pp. 598-601, 10/11/2014

This Conference Paper is brought to you for free and open access by the School of Electrical and Electronic Engineering at ARROW@TU Dublin. It has been accepted for inclusion in Conference papers by an authorized administrator of ARROW@TU Dublin. For more information, please contact arrow.admin@tudublin.ie, aisling.coyne@tudublin.ie,gerard.connolly@tudublin.ie. Funder: Irish Research Council

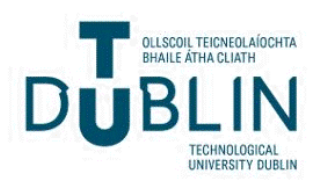




\section{Shaping the Axial-Ratio Footprint of Crossed-Dipole Antennas}

\author{
Adam Narbudowicz ${ }^{\mathrm{a}}$ \\ ${ }^{a}$ Antenna \& High Frequency Research Centre \\ Dublin Institute of Technology \\ Dublin, Ireland \\ \{adam.narbudowicz; max.ammann\}@dit.ie
}

\author{
Max J. Ammann
}

Janusz Przewocki ${ }^{\mathrm{b}}$

${ }^{\mathrm{b}}$ Institute of Mathematics

Polish Academy of Sciences

Warsaw, Poland

j.przewocki@gmail.com

\begin{abstract}
A method is proposed to adaptively change the axial-ratio beam of simple circularly-polarized antennas. It is shown, that by varying the phase shift between two orthogonal elements the axial-ratio beam shape can be dynamically adjusted. This allows control of the direction of minimum axial-ratio and the ability to increase or decrease the beamwidth in one of two planes. The method is intended for satellite navigation systems: it can improve the rejection of reflected signals in varying propagation conditions (e.g. urban canyons), while using simple and low-cost circularly-polarized antennas.
\end{abstract}

Keywords-circular polarization; axial ratio; GNSS; GPS

\section{Introduction}

Rejection of reflected signals is one of the key challenges in modern Global Navigation Satellite Systems (GNSS), as such signals cause significant position error. The use of circular polarization (CP) enables such rejection, however the antenna needs to have low axial-ratio (AR) to suppress the cross polarization. As the reflections may originate from various angles, antennas with a broad AR beamwidth are required. This has been achieved by many techniques, such as quadrifilar helix antennas [1], combination of bow-tie and dielectric dipoles [2], bird-nest-like dielectric resonator [3] or stacked patches [4]. However these designs are complex and expensive, not practical for use in small electronic devices.

In the literature, there is plenty of work reported on simple dual-fed circularly-polarized antennas, such as crossed dipoles [5] or dual-fed microstrip patches [6-7]. However most of the works (including [5-7]) propose to use a $\Delta_{\mathrm{ph}}=90^{\circ}$ phase shift between the two input ports. This is in order to achieve a $90^{\circ}$ phase shift between the two orthogonal components in the farfield. However, as will be demonstrated in this paper, feeding the antenna with $\Delta_{\mathrm{ph}} \neq 90^{\circ}$ will produce a circular polarization for directions other than normal to the antenna. This can be achieved by exploiting the mathematical properties of two vectors in 3-dimensional space and their projection onto a surrounding sphere. Consequently, a direction with optimum circular polarization (i.e. where $\mathrm{AR}=0 \mathrm{~dB}$ ) can be changed without changing the radiation pattern for co-polarization.

In this paper, we discuss a different approach to dual-fed circularly polarized antennas, such as crossed-dipoles or

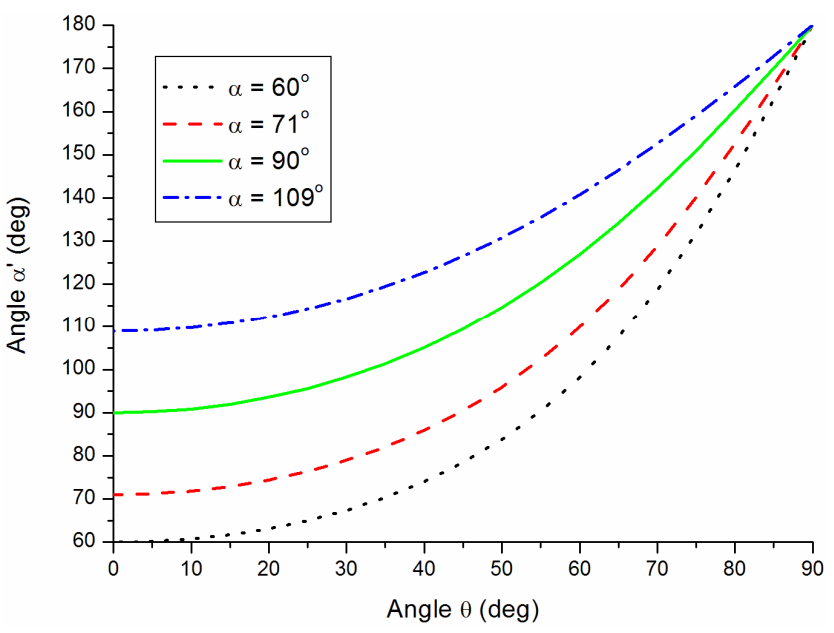

Fig. 1. Projected angle $\alpha^{\prime}$ for various angle $\theta$ in xz-plane, as described by (1)

patches. The proposed method adjusts the phase shift between the orthogonal components to expand the $3 \mathrm{~dB}$ AR beamwidth in one of two orthogonal planes. This allows dynamic adjustment of the AR pattern to changing conditions, such as to elongate the AR footprint in urban canyons for suppression of reflections from buildings. The method is intended for lowcost simple antenna elements and the phase variation can be easily controlled by an adjustable capacitor, switch or an integrated circuit. The proposed approach is general and can be used with dual-fed circularly-polarized antennas of any type.

\section{Theoretical background}

Let us investigate two intersecting vectors $u$ and $v$, of equal magnitude, lying in the $x y$-plane symmetrically with respect to the $x$ axis. The vectors form an angle $\alpha$ between themselves and an angle $\alpha / 2$ between each vector and the $x$-axis. Assume a circle in the $x z$-plane of radius $R>>u$, which originates at the point of intersection of $u$ and $v$. A projection of angle $\alpha$ onto a plane tangential to the circle in any given point can be described as: 


$$
\alpha^{\prime}=2 \arctan \left(\frac{\tan (\alpha / 2)}{\cos (\theta)}\right)
$$

where $\theta$ is the angle between the $z$-axis and the point in which the plane is tangential to the circle (i.e. corresponding to the polar angle in spherical coordinates). Fig. 1 visualizes eq. (1), showing the projected angle $\alpha^{\prime}$ as a function of $\theta$ for various angles $\alpha$. It can be seen, that the orthogonality for $\alpha=90^{\circ}$ will deteriorate with varying $\theta$. Also, for any $\alpha<90^{\circ}$ there exists at least one $\theta$, for which $\alpha^{\prime}=90^{\circ}$. For the border case of $\theta=90^{\circ}$ any angle $\alpha$ will be seen as $\alpha^{\prime}=180^{\circ}$. These principles drive the proposed AR pattern steering.

The commonly accepted definition of circular polarization requires the $\mathrm{AR}$ to be below $3 \mathrm{~dB}$, corresponding to a $15.5 \mathrm{~dB}$ cross-polarization level [8], which is sufficient for most civilian GNSS uses. Given equal amplitudes, the $3 \mathrm{~dB}$ limit at boresight $\left(\theta=0^{\circ}\right)$ is achieved when the phase difference at the dipole feeds $\Delta_{\mathrm{ph}}$ is up to $\pm 19^{\circ}$ distorted from $90^{\circ}$ (i.e. for $\Delta_{\mathrm{ph}}=71^{\circ}$ and $109^{\circ}$ ). For more demanding applications, an AR below $1 \mathrm{~dB}$ might be required, which corresponds to $24.8 \mathrm{~dB}$ of cross polarization. This can be achieved, when the phase difference at the dipole feeds $\Delta_{\mathrm{ph}}$ is up to $\pm 6^{\circ}$ distorted from $90^{\circ}$ (i.e. for $\Delta_{\mathrm{ph}}=84^{\circ}$ and $96^{\circ}$ ).

\section{Practical implementation}

Fig. 2 depicts a simple, state-of-the-art crossed-dipole antenna operating at GPS L1 frequency $1.575 \mathrm{GHz}$. The two arms are printed on the opposite sides of a dielectric substrate of $0.2 \mathrm{~mm}$ thickness and relative permittivity $\varepsilon_{\mathrm{r}}=3.5$. Each arm has a length $L=40.5 \mathrm{~mm}$ and width $W=3 \mathrm{~mm}$. The separation between the arms is $S=2 \mathrm{~mm}$. The circular dielectric has a diameter $D=90 \mathrm{~mm}$. Fig. 3 shows simulated $\mathrm{S}_{11}$ of the antenna, which is $-14 \mathrm{~dB}$ at a GPS L1 frequency of $1.575 \mathrm{GHz}$.

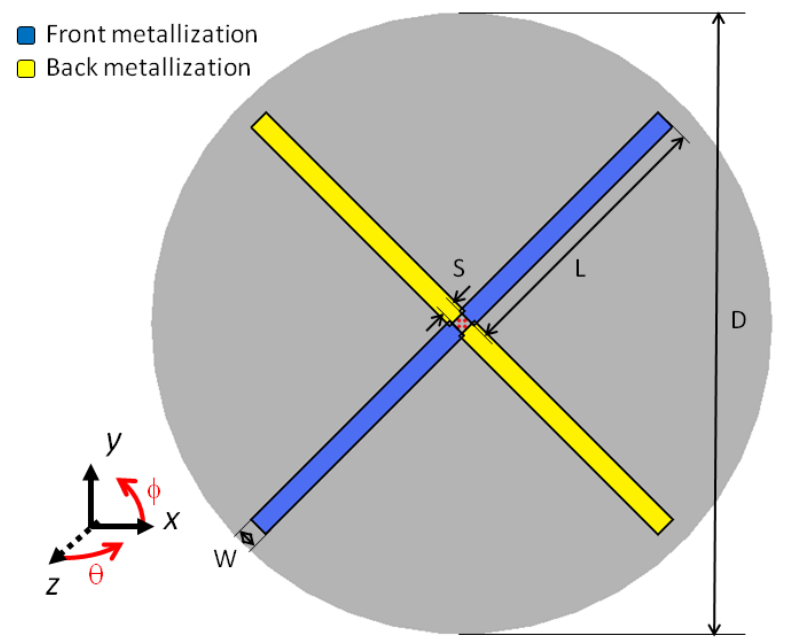

Fig. 2. The crossed dipole antenna used in the study.

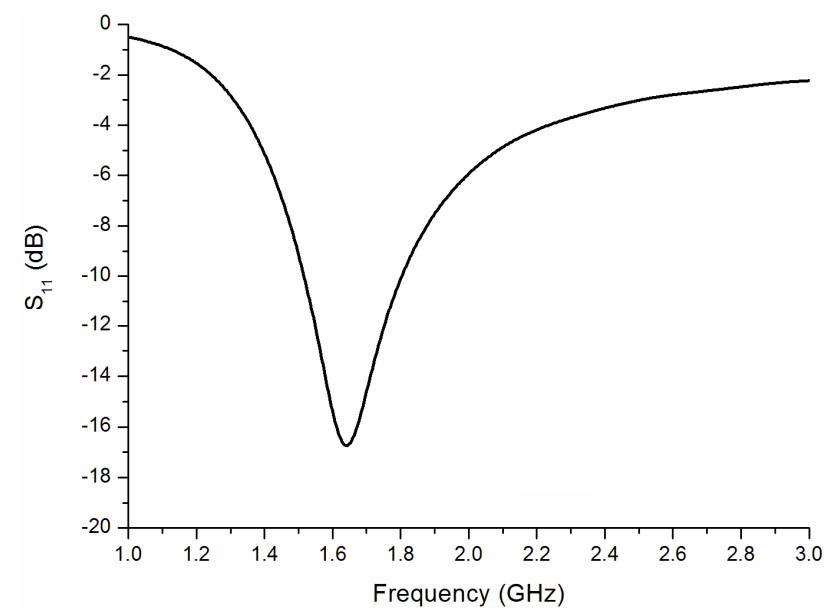

Fig. 3. Simulated $\mathrm{S}_{11}$ of the proposed antenna.

The two dipoles were fed with $\Delta_{\text {ph }}=71.7^{\circ}, 109.7^{\circ}$ and additionally $84.7^{\circ}$. The two first values were chosen to preserve the $\mathrm{AR} \leq 3 \mathrm{~dB}$ at boresight and $0.7^{\circ}$ was added to compensate for the $0.2 \mathrm{~mm}$ substrate thickness. The value of $\Delta_{\text {ph }}=84.7^{\circ}$ allows to preserve $\mathrm{AR} \leq 1 \mathrm{~dB}$ at boresight. Fig. 4 shows the simulated AR for the $\phi=0^{\circ}$ cut (xz-plane). According to (1) and Fig. 1 the optimum AR for $\Delta_{\text {ph }}=71.7^{\circ}$ (i.e. where $\alpha^{\prime}=90^{\circ}$ ) should be achieved for $\theta=44.5^{\circ}$. In practice, due to the $0.2 \mathrm{~mm}$ spacing between the dipoles, this value is seen in Fig. 4 for $\theta=45^{\circ}$. It can be also seen, that the $\mathrm{AR}$ at boresight is almost exactly $3 \mathrm{~dB}$, as expected from theoretical calculations. For $\Delta_{\mathrm{ph}}=71.7^{\circ}$ the $3 \mathrm{~dB}$ AR beamwidth in the $\phi=0^{\circ}$ cut ( $x z$-plane) extends to $\pm 60^{\circ}$, which is an improvement compared to the $3 \mathrm{~dB}$ beamwidth from $\pm 47^{\circ}$ offered by the traditionally used $\Delta_{\mathrm{ph}}=90^{\circ}$. The optimum AR is located halfway between the antenna zenith and the Earth's surface, which is more efficient at rejecting reflected signals from buildings. Furthermore, this angle can be flexibly modified by adjusting the phase shift. For $\Delta_{\mathrm{ph}}=109.7^{\circ}$ the $3 \mathrm{~dB}$ AR beamwidth in $x z$-plane is very narrow $\left( \pm 10^{\circ}\right)$ as the

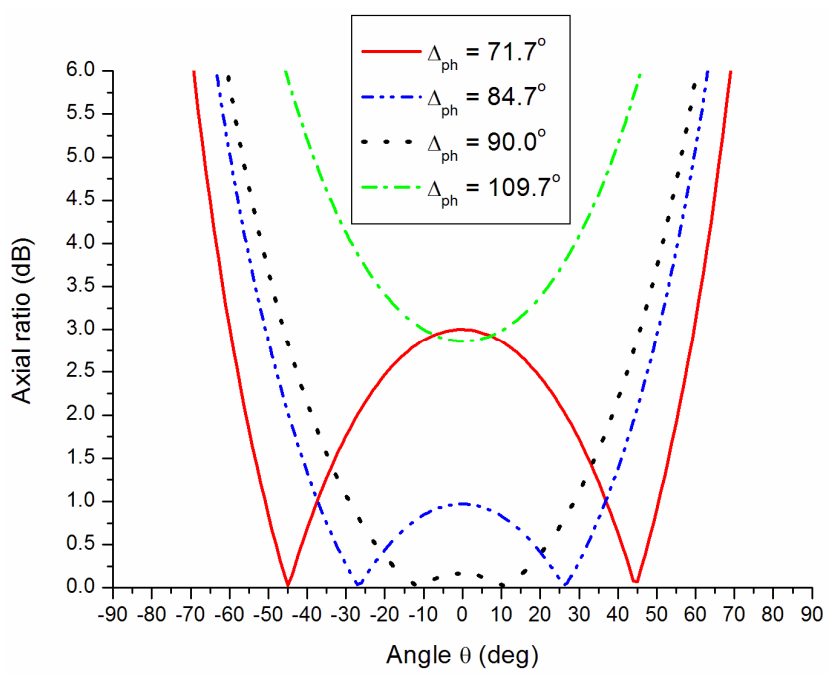

Fig. 4. Simulated AR as a function of $\theta$ in xz-plane 
best $\mathrm{AR}$ is in $y z$-plane $\left(\phi=90^{\circ}\right)$. For $\Delta_{\mathrm{ph}}=84.7^{\circ}$ the $\mathrm{AR} \leq 1$ $\mathrm{dB}$ beamwidth extends from $\pm 37^{\circ}$, which is an improvement as compared to traditionally used $90^{\circ}$ phase shift able to provide $\pm 29^{\circ}$.

Fig. 5 depicts the AR as a function of both $\theta$ and $\phi$ within a hemisphere. Angles with $\mathrm{AR} \leq 3 \mathrm{~dB}$ has been coloured with respect to the beam that produced it. It can be seen, that for $\Delta_{\mathrm{ph}}=71.7^{\circ}$ the AR beam (red and purple) is stretched in the $x z$-plane and constricted in $y z$-plane, whereas $\Delta_{\mathrm{ph}}=109.7^{\circ}$ (yellow and green) provides the opposite performance.

Fig. 6 and 7 show the simulated realized gains for right hand (RHCP) and left hand (LHCP) polarizations. Due to the specifics of crossed-dipole antenna, a counter-polarized beams are produced in opposite directions. It can be seen, that the optimum cross-polarization is seen for angles calculated in (1). It should be also noted, that the gain is independent from the phase shift used. For the three investigated configurations the variation of RHCP gain at boresight is below $0.15 \mathrm{dBic}$.

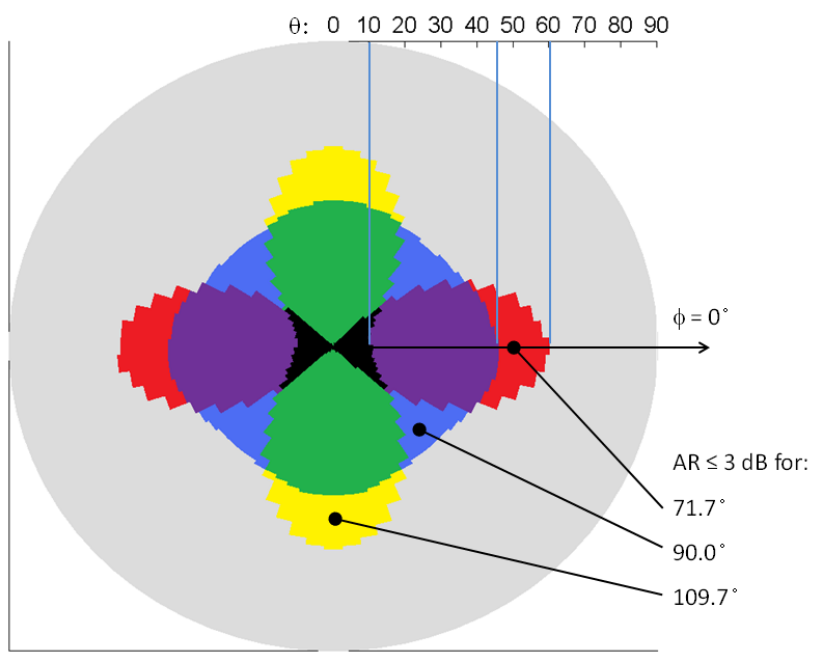

Fig. 5. Simulated axial ratio as a function of $\phi$ and $\theta$. Coloured areas mark $\mathrm{AR} \leq 3 \mathrm{~dB}$ for given feed.

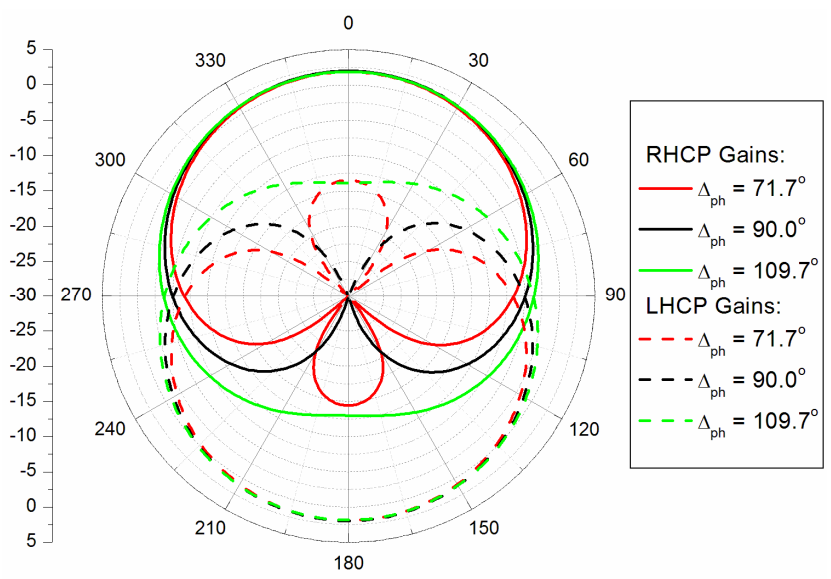

Fig. 6. Simulated realized gains in $x z$-plane of crossed dipole antenna for different values of phase shift $\Delta_{\mathrm{ph}}$. Please note, that the angle with lowest cross-polarizations corresponds to the lowest AR.

This work was funded by Science Foundation Ireland under Technology Innovation Development Award, grant number 13/TIDA/I2746 and by Irish Research Council under "Elevate" Fellowship, grant number ELEVATEPD/2014/79.

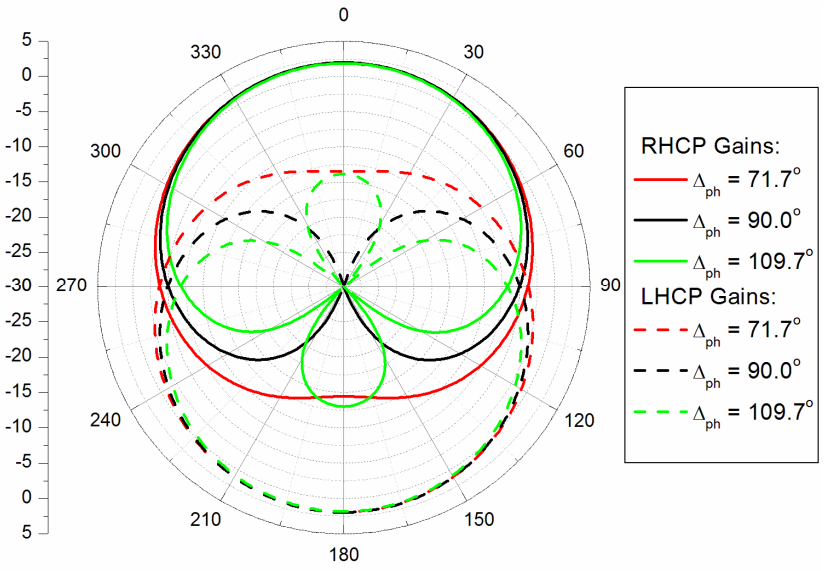

Fig. 7. Simulated realized gains in $y z$-plane of crossed-dipole antenna for different values of phase shift $\Delta_{\mathrm{ph}}$.

\section{Conclusion}

The proposed method allows reconfiguration of the direction of optimum $\mathrm{AR}$ and consequently re-shaping of the "footprint" where AR is below a given level. This reconfiguration does not affect antenna gain or co-polarized radiation pattern. The simulated results agree well with analytical predictions. Future work will prototype and measure the antenna, as well as to develop efficient method to incorporate it into GNSS receivers.

The method is demonstrated for GNSS application at 1.575 $\mathrm{GHz}$ and is intended to provide adaptive rejection of multipath signals. However the concept of steering AR is generic and can benefit other applications, e.g. it can be used to enhance privacy in CP modulated communication systems like [9].

\section{References}

[1] Y.-S. Wang, and S.-J. Chung, "A Miniature Quadrifilar Helix Antenna for Global Positioning Satellite Reception," IEEE Trans. on Antennas and Propagation, vol. 57 (12), pp. 3746-3751, Dec. 2009.

[2] K. M. Mak, and K. M. Luk, "A Circularly Polarized Antenna With Wide Axial Ratio Beamwidth," IEEE Trans. on Antennas and Propagation, vol. 57 (10), pp. 3309-3312, Oct. 2009.

[3] Y.-M. Pan, and K. W. Leung, "Wideband Circularly Polarized Dielectric Bird-Nest Antenna With Conical Radiation Pattern," IEEE Trans. on Antennas and Propagation, vol. 61 (2), pp. 563-570, Feb. 2013.

[4] X. L. Bao, and M. J. Ammann, "Dual-frequency dual circularlypolarised patch antenna with wide beamwidth," Electronics Letters, vol. 44 (21), pp. 1233-1234, Oct. 2008.

[5] J. W. Baik, K.-J. Lee, W.-S. Yoon, T.-H. Lee, and Y.-S. Kim, "Circularly polarised printed crossed dipole antennas with broadband axial ratio," Electronics Letters, vol. 44 (13), pp. 785-786, Jun. 2008.

[6] F. Ferrero, C. Luxey, R. Staraj, G. Jacquemod, M. Yedlin and V. Fusco, "A novel quad-polarization agile patch antenna," IEEE Trans. on Antennas and Propagation, vol. 57 (5), pp. 1563-1567, May 2009.

[7] A. Narbudowicz, Xiulong Bao, and M. J. Ammann, "Dual CircularlyPolarized Patch Antenna Using Even and Odd Feed-Line Modes," IEEE Trans. on Antennas and Propagation, vol. 61 (9), pp. 4828-4831, Sep. 2013.

[8] B. Y. Toh, R. Cahill, and V. F. Fusco, "Understanding and measuring circular polarization," IEEE Transactions on Education, vol. 46 (3), pp. 313-318, Aug. 2003. 
[9] M. Amin, S. Ahmed, V. Fusco, H. Cantu and T. Ratnarajah, "The Effect of Spatial Axial Ratio Variation on QPSK Modulation Encoded Using Orthogonal Circularly Polarized Signals," In Proc.: European
Conference on Wireless Technologies, pp. 62-65, Oct. 2007, Munich Gemrany. 